\title{
Cold-induced heat production preceding shivering
}

\author{
Anne M. J. van Ooijen ${ }^{1}$, Wouter D. van Marken Lichtenbelt ${ }^{1}$, Anton A. van Steenhoven ${ }^{2}$ and \\ Klaas R. Westerterp ${ }^{1}$ \\ ${ }^{1}$ Department of Human Biology, Maastricht University, Maastricht, The Netherlands \\ ${ }^{2}$ Department of Energy Technology, University of Technology, Eindhoven, The Netherlands
}

(Received 22 April 2004 - Revised 24 October 2004 - Accepted 27 October 2004)

\begin{abstract}
Individual changes in heat production and body temperature were studied in response to cold exposure, prior to shivering. The subjects ten women (seven men) were of normal weight, had a mean age of 23 (SD 3) years and average BMI 22.2 (SD 1.6) Kg/m². They were lying supine under thermoneutral conditions for $30 \mathrm{~min}$ and were subsequently exposed to air of $15^{\circ} \mathrm{C}$ until shivering occurred. Heat production was measured with a ventilated hood. Body composition was measured with underwater weighing and ${ }^{2} \mathrm{H}$ dilution. Body temperatures were measured with thermistors. Heat production during cold exposure prior to shivering increased and reached a plateau. Skin temperature decreased and did not reach a plateau during the test period. The non-shivering interval (NSI) ranged from 20 to $148 \mathrm{~min}$, was not related to body composition and was not significantly different between women (81 (SD 15) min) and men (84 (SD 34) min). NSI was negatively related to skin temperature $\left(r^{2} 0.44, P=0.004\right)$, and skin temperature was related to heat production $\left(r^{2} 0.39\right.$, $P=0.007)$. In conclusion, subjects with a relatively large heat production during cold exposure maintained a relatively high skin temperature but showed a short NSI, independent of differences in body composition.
\end{abstract}

Individual variation: Metabolism: Non-shivering interval: Temperature response

Upon entering a cooler environment, the body will adjust to restore heat balance. Heat balance can be restored by reducing heat loss or, on the other hand, increasing heat production. Behavioural aspects can intervene at both points: clothing can reduce heat loss and activity, and food intake can increase heat production. Even when behavioural aspects are taken into account, physiological reactions during resting conditions occur (Warwick \& Busby, 1990). The reduction of heat loss is established by vasoconstriction, which decreases heat transfer from core to the skin. This reduces the temperature of the skin and distal parts of the body (Frank et al. 1999).

On the other side of the balance, heat can be produced by several mechanisms, summarised as adaptive thermogenesis. Adaptive thermogenesis during resting conditions is subdivided into non-shivering thermogenesis (NST) and shivering thermogenesis (ST). Under severe cold circumstances, shivering starts soon but depends on the interplay between core temperature and skin temperature (Benzinger, 1969) and the change in skin temperature (Fiala et al. 2001). Shivering is a very efficient way to produce heat, and the peak shivering intensity can increase heat production up to five times the BMR (Eyolfson et al. 2001). The intensity of shivering is determined by the severity of cold exposure, the amount of fat-free mass (FFM), the subject's fitness (Eyolfson et al. 2001) and the rate of change of the skin temperature (Fiala et al. 2001). If cold exposure is not so severe, shivering does not start immediately (Vybiral et al. 2000). Although NST in humans preceding the onset of shivering, or in addition to shivering, has been observed before (Paolone \& Paolone, 1995; Vybiral et al. 2000), opinions on the existence and mechanisms involved are still divided (Nedergaard et al. 2001).

Although shivering has been intensively studied, the period preceding shivering has hardly been described (Vybiral et al. 2000). Nevertheless, the state of being exposed to mild cold without shivering is a condition occurring frequently in daily life. In an earlier investigation, we showed an increase in heat production in response to mild cold without shivering (van Ooijen et al. 2004) and that the relative magnitude of NST was related to the temperature response. This can have implications for energy balance and the risk of gaining weight (Dauncey, 1981; Ravussin et al. 1988). Insight into factors such as body composition, body shape, resting metabolic rate (RMR) and gender related to the cold-induced heat production can be of importance in the treatment and prevention of obesity (Van Marken Lichtenbelt \& Daanen, 2003).

In our previous investigation, factors determining NST could not yet be unravelled, possibly because heat production did not reach a plateau (van Ooijen et al. 2004). Therefore, in the present study, we used a protocol with mild cold exposure that was expected to last long enough to reach a metabolic plateau prior to shivering and was cold enough actually to induce shivering. This resulted in a well-defined non-shivering interval (NSI), making it possible to study individual differences in NST and the duration of NSI. It was hypothesised that subjects who cool down quickly have a relatively low skin temperature and a 
relatively short NSI. If subjects cool down fast, the shivering threshold is expected to be reached earlier. Furthermore, the question is addressed of whether subject characteristics (body fat, morphology, RMR) are related to NSI, NST or ST.

In summary, the aims of the present study were to investigate the following questions:

1. Does NST reach a plateau, or does it increase until shivering starts?

2. Does skin temperature reach a plateau, or does the temperature continue to drop until shivering starts?

3 . Is heat production related to the NSI?

4. Is the temperature response related to the NSI?

5. Are the temperature response and the response in heat production related?

6. Is it possible to identify subject characteristics (e.g. body composition or RMR) that are related to NST or ST?

\section{Materials and methods}

\section{Subjects}

Ten women and seven men participated in the study, previously approved by the Ethics Committee of Maastricht University. They volunteered and gave written consent to participate. All the subjects were healthy, were non-smokers and did not use any medication. Subjects had a mean age of 23 (SD) years, an average height of 178 (SD12) $\mathrm{cm}$ and average weight 66.9 (SD) $8.4 \mathrm{Kg}$ (Table 1).

\section{Design}

The experiment consisted of a cold air exposure test. Subjects were lying supine, their heads slightly tilted, on a stretcher, in $15^{\circ} \mathrm{C}$ air and covered with a duvet $\left(375 \mathrm{~g} / \mathrm{m}^{2}\right)$. This was the thermoneutral condition. Cold was induced after $30 \mathrm{~min}$ by removing the duvet. The test was terminated $30 \mathrm{~min}$ after the onset of shivering. The interval between the removal of the duvet and the onset of shivering was defined as the NSI. The interval between the onset of shivering and the termination of the test was defined as the shivering interval (SI). Metabolic rate, body temperature and electromyographs (EMG) were measured continuously. Body composition was determined on a separate visit within 1 week of the experiment.

Subjects arrived at the laboratory by car or public transport and were fasting for at least $4 \mathrm{~h}$ in order to avoid any effects of activity or diet. They were instructed not to perform strenuous activity the day before the experiment. The clothing had a total insulative value of $0.18 \mathrm{clo}\left(0.028 \mathrm{~m}^{2} \cdot{ }^{\circ} \mathrm{C} \mathrm{W}^{-1}\right)$ and consisted of

Table 1. Subject characteristics and non-shivering interval

\begin{tabular}{lccccc}
\hline & \multicolumn{2}{c}{ Men $(n 7)$} & & \multicolumn{2}{c}{ Women $(n$ 10) } \\
\cline { 2 - 3 } & Mean & SD & & Mean & SD \\
\hline Age (years) & 21 & 2 & & 24 & 3 \\
Height (m) & 1.90 & 0.06 & & 1.69 & 0.05 \\
Weight $(\mathrm{kg})$ & 74.8 & 4.1 & & 61.4 & 5.0 \\
BMl $\left(\mathrm{kg} / \mathrm{m}^{2}\right)$ & 20.8 & 1.6 & & 21.5 & 1.5 \\
Fat-free mass $(\mathrm{kg})$ & 65.7 & 4.5 & & 44.4 & 3.9 \\
Fat mass (\%) & 12.2 & 1.9 & & 27.5 & 6.6 \\
Non-shivering interval (min) & 81 & 15 & & 84 & 34 \\
\hline
\end{tabular}

shorts ( $0 \cdot 1$ clo), a singlet $(0.04$ clo $)$ and panties and a bra for women, and briefs for men ( 0.04 clo).

\section{Measurements and instrumentation}

Body composition was calculated using the three-compartment model according to Siri (1961). For this calculation, body density and total body water were determined using underwater weighing and ${ }^{2} \mathrm{H}$ dilution (Ellis, 2000). The latter was determined according to the Maastricht protocol (Westerterp et al. 1995).

$\mathrm{O}_{2}$ consumption and $\mathrm{CO}_{2}$ production were measured with an automated respiratory gas analyser using a ventilated hood system (Omnical; IDEE, Maastricht, The Netherlands). The equipment had recently been validated, and the gas analysers were calibrated before the experiments (Adriaens et al. 2003).

Heat production was calculated from these data according to Weir and are published in a classical article (Weir, 1990). RMR was defined as heat production in a thermoneutral environment, lying still and awake in a post-absorptive state. RMR was calculated as the average of $10 \mathrm{~min}$ with the lowest SD of the previous $20 \mathrm{~min}$ while the subject was covered with the duvet. NST was defined as the increase in heat production exceeding RMR that occurred during cooling and before the subjects started shivering. NST was calculated by subtracting RMR from the total heat production during this period. ST was defined as the heat production exceeding the sum of RMR and NST when the subjects were shivering, as registered by the EMG (see below). ST was calculated by subtracting RMR and NST from the total heat production during this period.

Rectal temperature was measured continuously by a thermistorprobe (YSI probes, series 402, Yellow Springs Instruments Co. Ltd., Ohio, USA) inserted $10 \mathrm{~cm}$ beyond the anal sphincter. Skin temperatures were measured by surface thermistors (YSI probes, series 409B, Yellow Springs Instruments Co. Ltd.) placed on the dorsal side of the hand, forearm, upper arm, chest, abdomen, lower back, anterior thigh, posterior calf and foot. Temperatures were registered and saved over $1 \mathrm{~min}$ intervals (Tiretherm, IDEE, Maastricht, The Netherlands).

Mean skin temperature was calculated according to Ramanathan (1964) and is hereafter designated 'skin temperature'. A weighted body temperature as a cue for thermoregulation, including rectal and skin temperatures, was calculated using the formula of Cunningham et al. (1978):

$$
T_{\text {body }}=T_{\text {core }}+0 \cdot 1\left(T_{\text {skin }}-33^{\circ} \mathrm{C}\right) \text {. }
$$

This measure combines the relative importance of skin and core temperatures for thermoregulatory adjustments.

Shivering was detected using an EMG (Tiretherm). EMG electrodes were placed $3 \mathrm{~cm}$ apart on the skin at the muscle pectoralis major (Tikuisis et al. 1991; Bell et al. 1992). This site was chosen because, in people possessing a normal amount of fat, shivering starts in the upper trunk region and propagates towards the extremities (Tikuisis et al. 1991). In addition, subjects were asked every $15 \mathrm{~min}$ whether they felt as if they were shivering, and the observer checked the subjects to see whether they were.

\section{Treatment of data}

Data are reported as means together with their standard deviations. Results were considered statistically significant when $P<0 \cdot 05$. The SPSS program, version 6.1 (SPSS Inc., Chicago, IL, USA) was used 
for statistical analyses. Linear regression analysis was used to study the relation between the metabolic and insulative response. If necessary, the analysis was corrected for body composition by adding FFM into the equation. Gender differences were tested by unpaired $t$ tests. The difference between men and women in terms of NSI was tested by the Mann-Whitney U test.

\section{Results}

Subjects

Subjects were healthy with a normal percentage of body fat (21.2 (SD 2.2) \%; Table 1). As can be expected, the men were significantly taller and heavier, and had a higher FFM $(\mathrm{kg})$ and a lower body fat percentage than the women.

Time course of heat production, muscle activity and temperature

A representative example of the progress of heat production, EMG signal and skin temperature is presented in Fig. 1. Fifteen out of the seventeen subjects showed similar trends. Three distinct phases can be recognised: thermoneutral, NSI and SI. Figure 1(A)

(A)

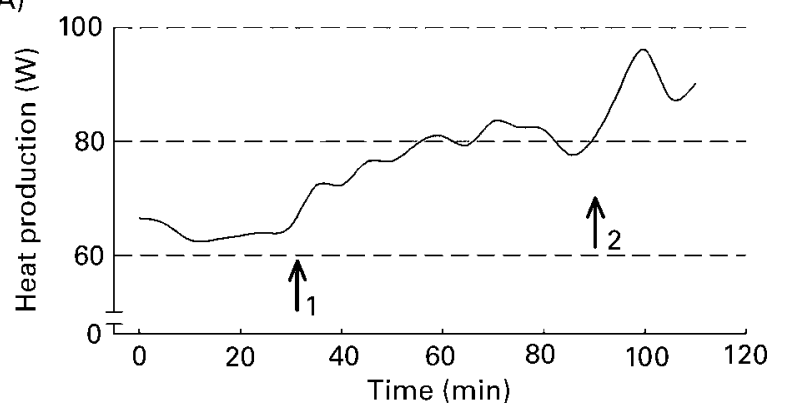

(B)

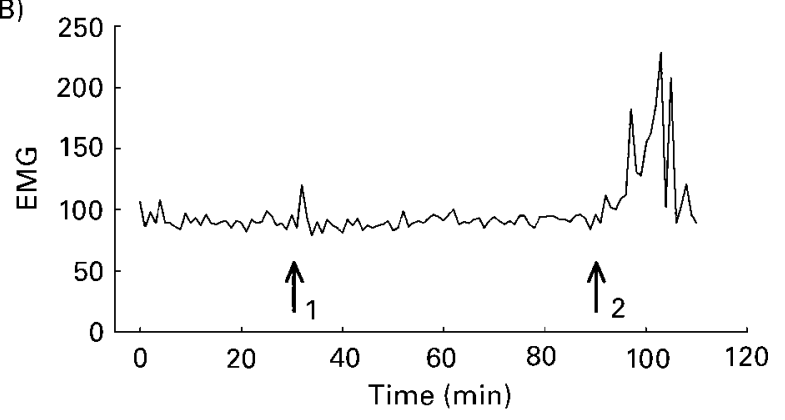

(C)

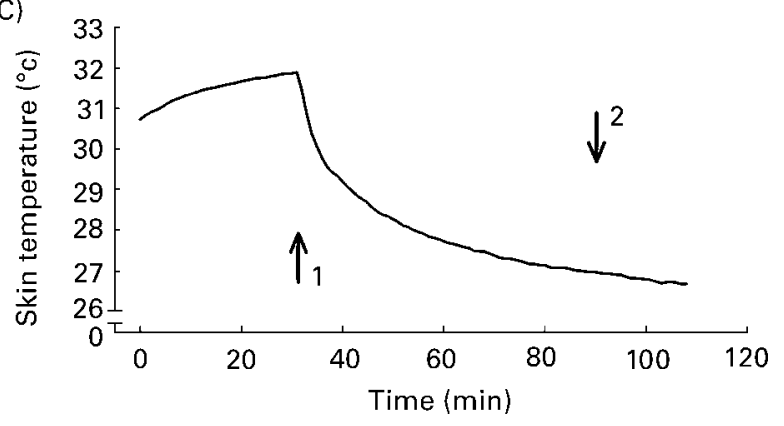

Fig. 1. (A) Typical pattern of heat production during the experiment. (B) Typical pattern of an electromyograph measurement during the experiment. (C) Typical pattern of skin temperature during the experiment. 1, duvet removed; 2 , onset of shivering. shows heat production throughout the experiment. During the thermoneutral period, the metabolic rate reached a plateau. During the NSI, heat production gradually increased and again reached a plateau. The slope of the regression line over the 15 min before shivering was not significant in eleven subjects, indicating that a plateau was reached in most subjects. For the total group, the change in heat production for this interval was not significantly different from zero $(P=0 \cdot 27)$. Heat production rose sharply after the onset of shivering. On average, heat production increased from 76.8 (SD 14.8) W during the thermoneutral phase to 87.7 (SD 19.2) W during the last 15 min preceding shivering $(P<0.001)$ and then to 96.3 (SD 21.6) W during the first $15 \mathrm{~min}$ of shivering $(P<0.001)$. The change in heat production during the NSI averaged $12 \%$, ranging from $-6 \%$ to $28 \%$. After the onset of shivering, heat production increased another $12 \%$ (range $-2 \%$ to $29 \%$ ).

The pattern of the EMG measurement of the same subject is shown in Fig. 1(B). During the thermoneutral interval and the NSI, the EMG signal showed a low amplitude. The increase in signal at the start of the NSI is an artefact caused by removal of the duvet. The SI is clearly marked by an increase in EMG amplitude.

The pattern of skin temperature of the same subject is shown in Fig. 1(C). This pattern is representative of all the subjects. During the thermoneutral interval, the skin temperature rose marginally. The start of the NSI is marked by a steep decline that tended to level off near the end of the experiment. Skin temperature did not, however, reach a plateau, either during the NSI or during the shivering interval. Mean skin temperature decreased from 32.5 (SD 0.9) ${ }^{\circ} \mathrm{C}$ during the thermoneutral phase to 27.2 (SD 1.4) ${ }^{\circ} \mathrm{C}$ at the onset of shivering, and then decreased further, to $27 \cdot 0$ (SD 1.4) ${ }^{\circ} \mathrm{C}$, during the SI (Table 2).

\section{Thermoneutral phase}

RMR was significantly higher in men than women $(P<0 \cdot 001)$. RMR was related to FFM (RMR (W) $=13.8+1.2$ FFM $(\mathrm{kg})$ $\left.P<0.001, r^{2} 0 \cdot 84\right)$. After correcting for FFM in multiple linear regression analysis, no significant difference remained between the genders.

Body temperatures during the thermoneutral phase are presented in Table 2.

Stepwise regression did not include skin temperature in the relation between FFM and RMR.

\section{Non-shivering interval}

The NSI ranged from 20 to 148 min (mean 83 (SD 15) min; Table 1). The NSI was not significantly different between men

Table 2. Metabolism and body temperature during the thermoneutral phase and at the onset of shivering

\begin{tabular}{|c|c|c|c|c|}
\hline & \multicolumn{2}{|c|}{$\begin{array}{c}\text { Thermoneutral } \\
\text { phase }\end{array}$} & \multicolumn{2}{|c|}{ Shivering onset } \\
\hline & Mean & SD & Mean & SD \\
\hline Heat production $(\mathrm{W})^{\star}$ & $76 \cdot 8$ & 14.8 & $87 \cdot 7$ & $19 \cdot 2$ \\
\hline Rectal temperature $\left({ }^{\circ} \mathrm{C}\right)^{*}$ & $37 \cdot 2$ & 0.2 & 36.9 & 0.3 \\
\hline Mean skin temperature $\left({ }^{\circ} \mathrm{C}\right)^{*}$ & 32.5 & 0.9 & $27 \cdot 2$ & 1.4 \\
\hline Body temperature $\left({ }^{\circ} \mathrm{C}\right)^{*}$ (weighted) & $37 \cdot 1$ & 0.2 & $36 \cdot 4$ & 0.4 \\
\hline
\end{tabular}

${ }^{\star} P<0.001$, thermoneutral phase versus shivering onset (paired $t$ test). 
and women $(P=0 \cdot 77)$. The range and standard deviation for both groups indicates large between-subject differences in response to cold.

The length of the NSI was not related to either FFM or fat mass. The duration of the NSI was negatively related to skin temperature during this interval $\left(r^{2} 0 \cdot 44, P=0 \cdot 004\right)$.

Mean skin temperature during the NSI was positively related to heat production during the NSI $\left(r^{2} 0.39, P=0.007\right)$. When heat production is corrected for FFM $(\mathrm{kg})$, the residuals of the relation between heat production (W) and FFM $(\mathrm{kg})$ are still related to mean skin temperature during the NSI $\left(r^{2} 0 \cdot 29, P=0.03\right)$.

The change in weighted body temperature was related to NST (W) $\left(r^{2} 0.39, P=0.007\right)$.

Furthermore, the rate of skin cooling (represented as the average change over time of skin temperature) was related to the mean skin temperature at the onset of shivering $\left(r^{2} 0.32\right.$, $P=0.02)$.

\section{Shivering interval}

During shivering, mean skin temperature was 27.9 (SD 1.3) ${ }^{\circ} \mathrm{C}$ and rectal temperature 36.9 (SD 0.3$){ }^{\circ} \mathrm{C}$. The increase in heat production during of shivering (ST) was not significantly related to FFM, fat mass, RMR or NST. The SI was probably too short to reach a balance, and these results will not be discussed any further below.

\section{Discussion}

In the present study, the change in heat production and the temperature response to cold were investigated in the period before shivering. The advantage of the present study protocol was a well-defined period between the start of cooling and the onset of shivering, allowing intersubject comparison of the NSI and temperature and metabolic responses during this interval. The results showed, on average, a significant increase in heat production induced by cold, in the absence of shivering. The interindividual variation in response is large. A recent validation of the equipment showed a mean within-subject cofficient of variation in resting heat production of $3.3 \%$ on separate days (Adriaens et al. 2003). It is thus unlikely that a change in heat production during our test larger than that is the result of a measurement or technical error.

A possible explanation for the decrease in heat production in some of the subjects is the $\mathrm{Q}_{10}$ effect. This means that, for a given decrease in temperature, the enzyme activity of the tissue will decrease as well, thereby decreasing heat production (Gordon, 2001). If the temperature of the subject's periphery decreases by $3{ }^{\circ} \mathrm{C}$, the $\mathrm{Q}_{10}$ effect can account for a $6 \%$ decrease in total body heat production. Subjects who do not, or insufficiently, increase local heat production will show a net decrease in total heat production. Even when local heat production induced by shivering is not enough to compensate for the total cooling of the body, the net result can be a decrease in heat production.

During the NSI, mean skin temperature was related to heat production, even after correcting for FFM. Subjects with a relatively high mean skin temperature are likely to lose more heat to the environment, although whether heat production during the NSI is related to heat loss has not been measured. In the case of shivering, such a relation has been described by
Tikuisis et al. (1991). They found that the intensity of shivering and the increase over the time of exposure was consistent with the increase in convective heat transfer coefficient, calculated from skin temperature and heat fluxes (Tikuisis et al. 1991). The contribution of NST is, however, small compared with ST, and when heat loss remains larger than heat production, shivering will be induced. In our data, a plateau was reached for heat production before the onset of shivering, while skin temperature was still decreasing. This indicates that heat balance was not reached during the NSI, so shivering started.

NSI and mean skin temperature during NSI were negatively related. In contrast to our expectations, subjects with a relatively high mean skin temperature had an early onset of shivering. A relatively high skin temperature means that a relatively large amount of heat is lost to the environment.

The present study shows that a high heat production is related to a relatively high mean skin temperature, and a relatively high mean skin temperature is related to a short NSI. This means indirectly that a relatively high heat production is related to a short NSI. On the other side of the balance, the lower the skin temperature during the NSI, the longer it takes to start shivering. This means, on the one hand, that less heat is dissipated to the environment and that further cooling is restricted, but, on the other, that these subjects tolerate relatively low peripheral body temperatures. This contradicts what we expected and what can be expected based on previous literature (Benzinger, 1969), namely that a low body temperature, rather than a relatively high body temperature as we found in the present study, results in an early onset of shivering. It should be noted that, in contrast with previous literature, the present study investigates interindividual differences. These results fit the concept of heat regulation described by Webb (1995) and Tikuisis (2003), as opposed to temperature regulation in a more classical sense.

The present study also showed that the rate of skin cooling during the NSI was related to skin temperature at the onset of shivering. Subjects with fast skin cooling had higher skin temperatures at the onset of shivering. This confirms that body temperature is not the only factor important in thermoregulation, change in body temperature or in the heat content of the body also being involved (Fiala et al. 2001). The subjects with fast skin cooling and a high mean skin temperature at the onset of shivering were also the subjects with a relatively high mean skin temperature during the NSI and a relatively short NSI.

The change in weighted body temperature during the NSI was related to NST, reflecting the fact that subjects with a relatively high heat production response showed a relatively small decrease in body temperature. This is in line with results from our previous study (van Ooijen et al. 2004) and others (Contaldo et al. 1986).

These interindividual differences can have the following consequences. Subjects responding to the cold with a relatively large decrease in temperature can be more energy efficient and could have a higher risk of gaining weight (Ravussin et al. 1988). A relatively high heat production combined with an early onset of shivering could be an advantage in preventing obesity through a change in energy balance. It should be noted that this reaction can be a disadvantage for survival in severe cold circumstances.

No relationship was found between RMR and NST. NST does not depend on the size of FFM but possibly on the morphology of the subject, on the activity of the tissues comprising the FFM of on genetic variation. 


\section{Conclusion}

To summarise, we found that in answer to the six questions posed (1) NST reaches a plateau during the NSI, whereas (2) body temperature does not. Both (3) heat production during cold exposure and (4) skin temperature were related to the duration of the NSI. (5) NST and the change in weighted body temperature were related, and (6) no subject characteristics were found that predicted NST or ST.

The present study shows that normal-weight subjects show interindividual variation in heat production in the response to cold. The relatively high response in heat production found in some subjects as opposed to those with a relatively large response in skin temperature can have consequences for energy balance and therefore for weight maintenance. This is confirmed by the result that the subjects with a relatively high heat production during the the NSI seem to start shivering at an earlier stage, in contrast with hypotheses based on previous studies within subjects. This implies that a high heat production before shivering results in a high overall heat production during cold exposure, including a relatively early onset of shivering.

\section{References}

Adriaens MP, Schoffelen PF \& Westerterp KR (2003) Intra-individual variation of basal metabolic rate and the influence of daily habitual physical activity before testing. Br J Nutr 90, 419-423.

Bell DG, Tikuisis P \& Jacobs I (1992) Relative intensity of muscular contraction during shivering. J Appl Physiol 72, 2336-2342.

Benzinger TH (1969) Heat regulation: homeostasis of central temperature in man. Physiol Rev 49, 671-759.

Contaldo F, Scalfi L, Coltordi A \& Lanzilli A (1986) Reduced coldinduced thermogenesis in familial human obesity. Klin Wochenschr 64, 177-180.

Cunningham DJ, Stolwijk JA \& Wenger CB (1978) Comparative thermoregulatory responses of resting men and women. J Appl Physiol 45, 908-915.

Dauncey MJ (1981) Influence of mild cold on $24 \mathrm{~h}$ energy expenditure, resting metabolism and diet-induced thermogenesis. $\mathrm{Br} J \mathrm{Nutr} \mathbf{4 5}$, $257-267$.

Ellis KJ (2000) Human body composition: in vivo methods. Physiol Rev 80, 649-680.

Eyolfson DA, Tikuisis P, Xu X, Weseen G \& Giesbrecht GG (2001) Measurement and prediction of peak shivering intensity in humans. Eur J Appl Physiol 84, 100-106.
Fiala D, Lomas KJ \& Stohrer M (2001) Computer prediction of human thermoregulatory and temperature responses to a wide range of environmental conditions. Int J Biometeorol 45, 143-159.

Frank SM, Raja SN, Bulcao CF \& Goldstein DS (1999) Relative contribution of core and cutaneous temperatures to thermal comfort and autonomic responses in humans. J Appl Physiol 86, 1588-1593.

Gordon CJ (2001) The therapeutic potential of regulated hypothermia. Emerg Med J 18, 81-89.

Nedergaard J, Golozoubova V, Matthias A, Asadi A, Jacobsson A \& Cannon B (2001) UCP1: the only protein able to mediate adaptive non-shivering thermogenesis and metabolic inefficiency. Biochim Biophys Acta 1504, 82-106.

Paolone VJ \& Paolone AM (1995) Thermogenesis during rest and exercise in cold air. Can J Physiol Pharmacol 73, 1149-1153.

Ramanathan NL (1964) A new weighting system for mean surface temperature of the human body. J Appl Physiol 19, 531-533.

Ravussin E, Lillioja S, Knowler WC, Christin L, Freymond D, Abbott WG, Howard BW \& Bogardus C (1988) Reduced rate of energy expenditure as a risk factor for body-weight gain. $N$ Engl J Med 318, $467-472$.

Siri WE (1961) Body composition from fluid spaces and density: analysis of methods. In Techniques for Measuring Body Composition, pp. 223-244 [J Brozek and A Henschel, editors]. Washington, DC: National Academy of Sciences.

Tikuisis P (2003) Heat balance precedes stabilization of body temperatures during cold water immersion. J Appl Physiol 95, 89-96.

Tikuisis P, Bell DG \& Jacobs I (1991) Shivering onset, metabolic response, and convective heat transfer during cold air exposure. $J$ Appl Physiol 70, 1996-2002.

Van Marken Lichtenbelt WD \& Daanen HA (2003) Cold-induced metabolism. Curr Opin Clin Nutr Metab Care 6, 469-475.

van Ooijen AM, van Marken Lichtenbelt WD, van Steenhoven AA \& Westerterp KR (2004) Seasonal changes in metabolic and temperature responses to cold air in humans. Physiol Behav 82, 545-553.

Vybiral S, Lesna I, Jansky L \& Zeman V (2000) Thermoregulation in winter swimmers and physiological significance of human catecholamine thermogenesis. Exp Physiol 85, 321-326.

Warwick PM \& Busby R (1990) Influence of mild cold on 24 h energy expenditure in 'normally' clothed adults. Br J Nutr 63, 481-488.

Webb P (1995) The physiology of heat regulation. Am J Physiol 268, R838-R850.

Weir JB (1990) New methods for calculating metabolic rate with special reference to protein metabolism. 1949 [classical article]. Nutrition 6, 213-221.

Westerterp KR, Wouters L \& van Marken Lichtenbelt WD (1995) The Maastricht protocol for the measurement of body composition and energy expenditure with labeled water. Obes Res 3, Suppl. 1, 49-57. 